\title{
Effect of Fluoxetine on Motor Recovery after Acute Haemorrhagic Stroke: A Randomized Trial
}

\author{
Irfan Ahmad Shah ${ }^{1 *}$, Ravouf P Asimi ${ }^{1}$, Yuman Kawoos ${ }^{2}$, Mushtaq A Wani ${ }^{1}$, Maqbool A Wani ${ }^{1}$ and Mansoor A Dar ${ }^{2}$ \\ ${ }^{1}$ Department of Neurology, Sheri-Kashmir Institute of Medical Sciences, Srinagar, India \\ ${ }^{2}$ Department of Psychiatry, Government medical college Srinagar, India
}

*Corresponding author: Irfan Ahmad Shah, Senior resident hostel, SKIMS, Soura, Srinagar, India, Tel: 91-9796957186; E-mail: irfanskims@gmail.com

Received date: February 26, 2016; Accepted date: March 29, 2016; Published date: April 05, 2016

Copyright: (c) 2016 Shah IA, et al. This is an open-access article distributed under the terms of the Creative Commons Attribution License, which permits unrestricted use, distribution, and reproduction in any medium, provided the original author and source are credited.

\begin{abstract}
Background: A few clinical trials have suggested that selective serotonin reuptake inhibitors (SSRI's) enhance motor recovery after stroke but no study has been done in haemorhagic stroke patients. We therefore aimed to investigate whether fluoxetine, an SSRI would enhance motor recovery in patients of haemorrrhagic stroke.

Methods: Patients who had haemorrhagic stroke with hemiplegia or hemiparesis and were aged between 18 years and 80 years were included in this double-blind, placebo-controlled trial. Patients were randomly assigned, in a $1: 1$ ratio to fluoxetine $(20 \mathrm{mg} / \mathrm{d}$, orally) or placebo for 3 months starting $5-10$ days after the onset of stroke. All patients also had routine physiotherapy. The primary outcome measure was the change on the FMMS between day 0 and day 90 after the start of the study drug.
\end{abstract}

Results: A total of 89 patients were assigned to fluoxetine $(n=45)$ or placebo $(n=44)$, group, and 84 were finally included in the analysis (42 vs 42) after 5 patients lost to follow up. Mean FMMS score improved significantly greater at day 90 in the fluoxetine group (mean 35.64 points) than in the placebo group (23.60 points; $p=0.001)$.

Conclusion: Use of fluoxetine in patients of haemorrhagic stroke in early post stroke period added to physiotherapy increased recovery in motor deficits at 3 months.

Keywords: Stroke; Motor recovery; Fluoxetine; Haemorrhagic stroke

\section{Introduction}

Stroke is a global health problem. It is the second commonest cause of death and fourth leading cause of disability worldwide. Each year, stroke affects about 16 million people for the first time and causes about 5.7 million deaths [1]. Globally, 12.6 million people have moderate to severe disability following stroke and of this, 8.9 million are from low and middle income countries. Moreover, survivors of stroke account for about 51 million disability-adjusted life years (DALYs) [2,3].

Increasing evidence supports the assumption that the brain is plastic and improvements can be expected even after permanent injuries [4-6]. Clinical trials on role of pharmacotherapy in functional recovery after stroke have mainly involved three classes of drugs; noradrenergic agonists, serotonergic agonists and the dopamine agonists. In animal studies, multiple, potentially beneficial effects of SSRIs have been demonstrated in both normal and diseased brains [7-9].

In human studies also, multiple trials using Selective Serotonin Reuptake Inhibitors (SSRI's) have shown a positive effect on poststroke motor recovery. Dam et al. showed that fluoxetine may improve functional outcome compared to maprotiline or placebo [10]. Acler et al. showed using tandem mass spectrometry that citalopram improves cortical excitability and NIHSS score [11]. Pariente et al. demonstrated using functional MRI technique that fluoxetine modulates motor performance and cerebral activation of patients recovering from stroke [12]. Chollet et al. showed that fluoxetine significantly improved motor score in stroke patients at 3 months [13]. A recent Cochrane database review concluded that there were statistically significant benefits of SSRI's in reducing dependency and disability after stroke [14].

Haemorrhagic stroke constitutes about 7 to $27 \%$ of all strokes and has higher incidence in Kashmir province of India and in parts of Southeast Asia and China $[15,16]$. Keeping in view the above evidence suggesting a role of serotonergic drugs in stroke rehabilitation, we aimed to test whether a 3-month treatment with fluoxetine would enhance motor recovery in patients of haemorrhagic stroke.

\section{Materials and Methods}

\section{Design}

It was a hospital based prospective randomized controlled clinical study conducted over a period of one year from January 2014 to January 2015. The study was conducted in a tertiary care centre in northern India. The subjects for the study were selected from the patients admitted in the stroke care ward. The diagnosis of stroke was made on the basis of history, examination and CT scan findings of acute haemorrhage in the region of putamen or thalamus. Patients with acute hemorrhagic stroke within the past 5-10 days that caused hemiparesis or hemiplegia with age between 18 years and 80 years and Fugl Meyer motor scale (FMMS) scores of 55 or less at baseline were included. 
Page 2 of 5

Patients were excluded from study if they had severe post-stroke disability \{National Institute Of Health Scale score (NIHSS) $>20\}$, pregnancy or any major diseases that would prevent follow-up, clinically diagnosed depression or Montgomery Asberg depression rating scale (MADRS) score of more than 19 or Patients taking neuroleptic drugs, antidepressants, or benzodiazepines during the month before inclusion were also excluded as were subjects with substantial pre-existing disability that could affect assessment like residual deficits from a previous stroke $[17,18]$.

The study was approved by the institute ethical committee of SheriKashmir Institute of medical sciences. All patients provided written informed consent for participation in the study.

\section{Randomization, intervention and outcome measures}

A baseline assessment of various study parameters was done using the designated scales i.e. Fugl-Meyer motor scale (FMMS), Modified Rankin Scale (mRS), and Montgomery Asberg depression rating scales. Afterwards patients were allocated through randomization on 1:1 basis to treatment group and control group. Matching was done on 1:1 basis for age, sex and severity of stroke. Treatment group received fluoxetine in capsule form initially started at a dose of $10 \mathrm{mg}$ and later increased to $20 \mathrm{mg}$ after a period of 1 week. Similar dosage of fluoxetine has been used in most of the studies $[10,12,13]$. The placebo which was an inert capsule similar to that of fluoxetine was given to the control group.

All patients irrespective of the group also received routine stroke care during the trial period. Patients, attendants, study staff, and investigators were masked to treatment allocation. Patients were advised to report any side effect of the drug on phone or through hospital visit. Patients were followed for a minimum of 2 visits during the study period, one at 4 to 6 weeks and second at the end of treatment. On first visit, routine check-up was done and patient was enquired about possible adverse effects. Also compliance was checked and assured. On last visit, along with above parameters, reassessment was done using the various scales.

The primary outcome of the study was the mean change in Fugl Meyer Motor Scale (FMMS) score between inclusion (day 0) and day 90. The treatment duration of 90 days was selected as most of the improvement after stroke occurs in the initial 3 months and similar trial period was undertaken by other studies [13].

FMMS is a validated scale that has been widely used for motor assessment after stroke and has excellent intra-rater and inter-rater reliability and validity. The motor score has a range of 0 to 100 , with 66 points for the upper limb and 34 points for the lower limb; higher score indicating better motor power. Each item is rated as not (0), partly (1), or fully (2) performed. Secondary measures included change in Modified Rankin Scale (mRS) score and side effect profile of the drug [19].

\section{Data Analysis}

The statistical analysis was performed using SPSS version 20 software. Categorical variables were compared employing nonparametric tests (chi-square, Fischer exact test) whereas continuous variables were compared by using student's $t$ test. Values are expressed as mean $\pm \mathrm{SD}$ and $\mathrm{p}$ value $<0.05$ was considered significant. The sample size was calculated with the help of statistical software $G$ power=3.1.5 for medium effect size, i.e. effect size $d=0.75$.
The mean values for experimental group $(36.4 \pm 21.3)$ and for control group $(21.9 \pm 16.9)$ have been considered. The required sample size was 50 for each group by considering the above values with level of significance $B=5 \%$ and power of study $=95 \%$. A total of 42 patients were however finally enrolled in each group and the power of the study was more than $90 \%$.

\section{Results}

A total of 84 patients were finally analyzed for study variables. Majority of the patients in our study were in the age group of 50-60 and 60-70 years. The two groups were well balanced in terms of baseline and demographic characteristics and stroke severity. Males were slightly more in cases than controls. The difference was due to attrition of more females from cases. However matching for sex was done at the beginning of the study on 1:1 basis and hence did not have significant influence on results. Mean age of cases was higher (59.93 year) than controls (57.62 years).

\begin{tabular}{|c|c|c|}
\hline & Fluoxetine $(n=42)$ & Placebo $(n=42)$ \\
\hline Mean Age (years) & $59.93 \pm 8.400$ & $57.62 \pm 8.115$ \\
\hline Male & $29(69 \%)$ & $25(59.5 \%)$ \\
\hline \multicolumn{3}{|l|}{ Vascular risk factors } \\
\hline Diabetes & $7(16.7 \%)$ & $6(14.3 \%)$ \\
\hline Hypertension & $39(92.9 \%)$ & $42(100 \%)$ \\
\hline Dyslipidaemia & $7(16.7 \%)$ & $6(14.3 \%)$ \\
\hline Current smoker & $17(40.5 \%)$ & $13(31 \%)$ \\
\hline \multicolumn{3}{|l|}{ Haemorrhage characteristics } \\
\hline \multicolumn{3}{|l|}{ location } \\
\hline putamen & $22(52.4 \%)$ & $23(54.8 \%)$ \\
\hline thalamus & $20(47.6 \%)$ & $19(45.2 \%)$ \\
\hline Average volume $(\mathrm{ml})$ & $35.12 \pm 12.272$ & $36.21 \pm 12.869$ \\
\hline \multicolumn{3}{|l|}{ Baseline stroke severity } \\
\hline Total FMMS score & $18.31 \pm 2.509$ & $19.40 \pm 2.548$ \\
\hline Upper limb FMMS score & $7.45 \pm 1.173$ & $8.19 \pm 1.311$ \\
\hline Lower limb FMMS score & $10.86 \pm 1.671$ & $11.21 \pm 1.415$ \\
\hline NIHSS score & $13.60 \pm 2.275$ & $13.48 \pm 2.200$ \\
\hline \multicolumn{3}{|l|}{ Modified Rankin scale score } \\
\hline $0-2$ & $0(0.00 \%)$ & $0(0.00 \%)$ \\
\hline 3 & $2(4.76 \%)$ & $2(4.76 \%)$ \\
\hline 4 & $17(40.48 \%)$ & $20(47.6 \%)$ \\
\hline 5 & $23(54.76 \%)$ & $20(47.6 \%)$ \\
\hline MADRS score & $4.38 \pm 3.060$ & $4.55 \pm 2.568$ \\
\hline $\begin{array}{l}\text { Time from stroke to treatment } \\
\text { (days) }\end{array}$ & $9 \cdot 1(1 \cdot 6)$ & $9.3(1 \cdot 6)$ \\
\hline
\end{tabular}

Table 1: Baseline characteristics. 
Page 3 of 5

Treatment compliance was similar in the two groups. The mean number of capsules taken in the fluoxetine group was $85.8 \pm 6.2$ while in placebo group, it was $86.2 \pm 5.4$ capsules. Time from stroke to treatment was $9.1 \pm 1.0$ days in treatment arm and $9.3 \pm 0.8$ days in control arm $(\mathrm{p}=0.711)$.

The major risk factors for stroke like diabetes, smoking and dyslipidemia were similar in the two arms. Hypertension however was more in placebo group.

NIHSS, mRS, and MADRS mean scores did not differ significantly in the two groups at the beginning. Putamenal haemorrhage was more common than thalamic haemorrhage in both the groups. The mean volume of haemorrhage in cases was $35.12 \mathrm{ml}$ and in controls was $36.21 \mathrm{ml}$ with insignificant difference.

The baseline total Fugl Meyer motor score was higher in controls than cases. The difference was not statistically significant.

None of the patients was functionally independent $($ MRS $\leq 2)$ at the time of inclusion in the study. Mean baseline MADRS score was almost similar in the two groups (Table 1).

At 90 days, the mean total FMMS score was 53.95 in cases and 44.00 among controls. The difference was statistically significant. The mean change in both upper limb score and lower limb score showed statistical significance (Table 2).

\begin{tabular}{|l|l|l|l|}
\hline Day 90 FMMS Score & $\begin{array}{l}\text { Cases } \\
\text { (Mean } \pm \text { Sd) }\end{array}$ & $\begin{array}{l}\text { Controls } \\
\text { (Mean } \pm \text { Sd) }\end{array}$ & P Value \\
\hline UPPER LIMB & $30.76 \pm 2.835$ & $22.57 \pm 2.661$ & 0.001 \\
\hline LOWER LIMB & $23.19 \pm 2.616$ & $20.43 \pm 2.605$ & 0.015 \\
\hline TOTAL & $53.95 \pm 4.417$ & $43.00 \pm 4.384$ & 0.001 \\
\hline
\end{tabular}

Table 2: FMMS score at 90 days.

The mean change in total FMMS score was 35.64 among cases and 23.60 among controls with a statistically significant difference. The mean change in upper limb score was 23.31 among cases and 14.38 in controls and the difference was significant. The change in lower limb score (12.33 vs 9.12) was also significant (Table 3 ).

\begin{tabular}{|l|l|l|l|l|}
\hline & Cases & Controls & Difference & P value \\
\hline Mean Total FMMS & 35.64 & 23.60 & 12.04 & 0.001 \\
\hline Mean Upper limb FMMS & 23.31 & 14.38 & 8.93 & 0.001 \\
\hline Mean Lower limb FMMS & 12.33 & 09.12 & 3.21 & 0.011 \\
\hline
\end{tabular}

Table 3: Mean change in FMMS score from day 0 to day 90.

Independence in activities of daily life, measured by use of mRS, improved during treatment in both groups. The mean change in MRS score was not significant between the two arms.

However more people had a score of 0-2 in cases i.e. more people were functionally independent at 90 days.

The main adverse events in our study were insomnia, epigastric pain, nausea, anxiety, diarrhoea and palpitations. Among these epigastric pain, nausea and insomnia were significantly higher in the group receiving fluoxetine than in the placebo group (Table 4).

\begin{tabular}{|l|l|l|l|l|}
\hline & Cases & $\%$ & Controls & $\%$ \\
\hline Epigastric pain & 7 & 16.67 & 3 & 7.14 \\
\hline Nausea & 6 & 14.29 & 4 & 9.52 \\
\hline Insomnia & 8 & 19.00 & 5 & 11.90 \\
\hline Anxiety & 5 & 11.90 & 4 & 9.52 \\
\hline Diarrhoea & 4 & 9.52 & 3 & 7.14 \\
\hline Palpitations & 3 & 7.14 & 2 & 4.76 \\
\hline
\end{tabular}

Table 4: Adverse effects.

\section{Discussion}

Our study showed a beneficial effect on motor recovery in patients of acute haemorrhagic stroke who were treated with fluoxetine for a period of 3 months. The positive effect which was primarily assessed by a change in mean Fugl Meyer motor score (FMMS) from day zero to day 90 in the treatment and control groups, was significantly higher in patients receiving fluoxetine than in the control arm. In the recovery period following a stroke, improvement in motor scores generally occurs in almost all patients who survive the initial insult. However in our cohort the improvement in motor score in the treatment arm was substantially higher than control arm which indicates that the drug hastens motor recovery. The change was noticeable in the Fugl Meyer sub scores for upper and lower limbs also, which shows that the drug affects the overall motor function of the afflicted side of the body. Also the mRS scores showed that more patients were functionally independent (score 0-2) in the fluoxetine group than in the placebo group at the end of the study.

Few human studies testing the effect of SSRI's on stroke recovery and brain function have been reported and all of these trials have suggested a positive role of these drugs in post stroke functional outcome. However most of these studies have had small patient number and highlight the need for further research in this area. $[10,11,20,21]$ Earlier studies suggested that treatment of post stroke depression after stroke is associated with greater recovery in activities of daily living function [22]. Later studies showed that the effect was significant in non-depressed patients as well and even single doses of SSRI's influenced brain motor activation which could not be explained by the antidepressant effects. $[23,24]$. Most of such studies were done in patients of ischemic stroke, and our study was the first of its kind to be done in patients of haemorrhagic stroke exclusively. Another multicentric randomized trial (FMRICH trial) is presently undergoing to test the action of selective serotonin reuptake inhibitors (SSRI's) in motor recovery in haemorrhagic stroke patients and the results are expected by 2017 [25].

A study by Pariente et al. using functional MRI in 8 patients showed that a single dose of fluoxetine modulated cerebral sensory-motor activation in patients of pure motor hemiparesis [12]. Loubinoux and colleagues showed that paroxetine dose dependently modulates activity of entire motor pathway and reorganized motor processing [26]. In a placebo controlled, double blind study Zittel et al. evaluated the effect of single dose of citalopram on 8 patients of chronic stroke and found that dexterity was significantly improved [27]. The FLAME trial has shown promising results in the improvement in motor 
function of hemiparetic stroke patients. Our study showed higher values in motor scores at 3 months in both the cases and controls compared to the participants of the FLAME trial. The reason could be that we studied motor recovery after haemorrhagic stroke, while as the FLAME trial was done on ischemic stroke patients only. Also in our study the baseline FMMS values were higher indicating a slightly better motor function at day zero, than the FLAME trial, which could explain better motor scores at 90 days [13].

In animal studies, multiple, potentially beneficial effects of SSRIs have been demonstrated in both normal and diseased brains. First, SSRIs have a neurotrophic effect. Neurotrophins control neural plasticity, i.e. ability to change, or easily changed or shaped, in adults; regulate synaptic activity and neurotransmitter synthesis, and are essential for the regeneration of nerves [28]. SSRIs increase neurogenesis and expression of neurotrophic or growth factors in the adult hippocampus [8] and this is likely to account for the behavioural benefits of antidepressants in animals [29]. Importantly, several studies have shown that migration of new neurones to damaged areas of brain may occur [30] and that neurogenesis can also occur within areas of damaged brain [31]. Second, fluoxetine may have a neuroprotective effect. In animals, there may be several mechanisms for neuroprotective effects of SSRIs, such as reducing inflammation (e.g. repression of microglia activation) [32] and by enhancement of specific protein expression (hypoxia inducible factor-1 alpha) [33]. Third, SSRIs can indirectly affect an important hormonal system in the body, the adrenergic system, through up-regulation of betal receptors [34]. SSRIs may also improve recovery after stroke simply through their effect on preventing or abating depression and through increasing alertness and alleviating fatigue.

The treatment was relatively well tolerated and there were only few side effects of drug treatment. In particular dyspepsia and insomnia were significant complaints but mostly settled within some days. However, none of the complications needed hospitalization. Overall fluoxetine is a well-tolerated and time tested drug and its cost is also reasonable. Our study had quite a few limitations as well. First the sample size was small and patients were recruited from a single hospital .Those who were included were selected using a strict criteria and hence the studied patients do not fully represent the general stroke population. The maximum dose of fluoxetine used was $20 \mathrm{mg}$; while as higher doses could have been used for more explicit results. Also patients were not followed beyond 3 months and hence we don't know whether the effect of drug persisted or not.

The results of our study and the review of related studies suggests that at present fluoxetine and related drugs are the only pharmaceutical agents that may favourably influence the course of motor recovery after stroke and as such should generate discussions among stroke care specialists all over the world [35]. The drug being safe can be tried on individual basis and more dedicated research needs to be undertaken in this area.

\section{References}

1. Strong K, Mathers C, Bonita R (2007) Preventing stroke: saving lives around the world. Lancet Neurol 6: 182-187.

2. Fisher M, Norrving B (2011) 1st global conference on healthy lifestyles and non-communicable diseases control. Moscow, April 28-29.

3. Steinwachs DM, Collins-Nakai RL, Cohn LH, Garson A, Wolk MJ (2000) The future of cardiology: utilization and costs of care. J Am Coll Cardiol 35: 91B-98B.
4. Steultjens EM, Dekker J, Bouter LM, van de Nes JC, Cup EH, et al. (2003) Occupational therapy for stroke patients: a systematic review. Stroke 34: 676-687.

5. Liepert J, Miltner WH, Bauder H, Sommer M, Dettmers C, et al. (1998) Motor cortex plasticity during constraint-induced movement therapy in stroke patients. Neurosci Lett 250: 5-8.

6. Liepert J, Graef S, Uhde I, Leidner O, Weiller C (2000) Training-induced changes of motor cortex representations in stroke patients. Acta Neurol Scand 101: 321-326.

7. Ming GL, Song H (2005) Adult neurogenesis in the mammalian central nervous system. Annu Rev Neurosci 28: 223-250.

8. Schmidt HD, Duman RS (2007) The role of neurotrophic factors in adult hippocampal neurogenesis, antidepressant treatments and animal models of depressive-like behaviour. BehaviouralPharmacolog 18: 391-418.

9. Wiltrout C, Lang B, Yan Y, Dempsey RJ, Vemuganti R (2007) Repairing brain after stroke: a review on post-ischemic neurogenesis. Neurochem Int 50: 1028-1041.

10. Dam M, Tonin P, De Boni A, Pizzolato G, Casson S, et al. (1996) Effects of fluoxetine and maprotiline on functional recovery in poststroke hemiplegic patients undergoing rehabilitation therapy. Stroke 27: 1211-1214.

11. Acler M, Robol E, Fiaschi A, Manganotti P (2009) A double blind placebo RCT to investigate the effects of serotonergic modulation on brain excitability and motor recovery in stroke patients. J Neurol 256: 1152-1158.

12. Pariente J, Loubinoux I, Carel C, Albucher JF, Leger A, et al. (2001) Fluoxetine modulates motor performance and cerebral activation of patients recovering from stroke. Ann Neurol 50: 718-729.

13. Chollet F, Tardy J, Albucher JF, Thalamas C, Berard E, et al. (2011) Fluoxetine for motor recovery after acute ischaemic stroke (FLAME): a randomised placebo-controlled trial Lancet Neurol 10: 123-130.

14. Mead GE, Hsieh CF, Lee R, Kutlubaev MA, Claxton A, et al. (2012) Selective serotonin reuptake inhibitors (SSRIs) for stroke recovery. Cochrane Database Syst Rev 11: CD009286.

15. Wei JW, Arima H, Huang Y (2010) Variation in the frequency of intracerebral haemorrhage and ischaemic stroke in China: a national, multicentre, hospital register study; China QUEST Investigators. Cerebrovasc Dis 29: 321-327.

16. Chen D, Román GC, Wu GX, Wu ZS, Yao CH, et al. (1992) Stroke in China (Sino-MONICA-Beijing study) 1984-1986. Neuroepidemiology 11: 15-23.

17. Fugl-Meyer AR, Jaasko L, Leyman I, Olsson S, Steglind S (1975) The post stroke hemiplegic patient. I. A method for evaluation of physical performance. Scand J Rehabil Med 7: 13-31.

18. (2003) NIH Sroke Scale.

19. Bonita R, Beaglehole R (1988) Recovery of motor function after stroke. Stroke 19: 1497-1500.

20. Miyai I, Reding MJ (1998) Effects of Antidepressants on Functional Recovery Following Stroke: A Double-Blind Study. Neurorehabil Neural Repair 12: 5-13.

21. Robinson RG, Schultz SK, Castillo C, Kopel T, Kosier JT, et al. (2000) Nortriptyline versus fluoxetine in the treatment of depression and in short-term recovery after stroke: a placebo-controlled, double-blind study. Am J Psychiatry 157: 351-359.

22. Chemerinski E, Robinson RG, Kosier JT (2001) Improved recovery in activities of daily living associated with remission of poststroke depression. Stroke 32: 113-117.

23. Berends HI, Nijlant J, van Putten M, Movig KL, IJzerman MJ (2009) Single dose of fluoxetine increases muscle activation in chronic stroke patients. Clin Neuropharmacol 32: 1-5.

24. Zittel S, Weiller C, Liepert J (2007) Reboxetine improves motor function in chronic stroke. A pilot study. J Neurol 254: 197-201.

25. Marquez-Romero JM, Arauz A, Ruiz-Sandoval JL, Ede LCE, HuertaFranco MR, et al. (2013) Fluoxetine for motor recovery after acute 
Citation: Shah IA, Asimi RP, Kawoos Y, Wani MA, Wani MA, et al. (2016) Effect of Fluoxetine on Motor Recovery after Acute Haemorrhagic Stroke: A Randomized Trial. J Neurol Neurophysiol 7: 364. doi:10.4172/2155-9562.1000364

Page 5 of 5

intracerebral hemorrhage (FMRICH): study protocol for a randomized double-blind, placebo controlled, multi-center trial. Trials 19: 14-77.

26. Loubinoux I, Pariente J, Boulanouar K, Carel C, Manelfe C, et al. (2002) A single dose of the serotonin neurotransmission agonist paroxetine enhances motor output: double-blind, placebo-controlled, fMRI study in healthy subjects. Neuroimage 15: 26-36.

27. Zittel S, Weiller C, Liepert J (2008) Citalopram improves dexterity in chronic stroke patients. Neurorehabil Neural Repair 22: 311-314.

28. Lang UE, Jockers-Scherubl MC, Hellweg R (2004) State of the art of the neurotrophin hypothesis in psychiatric disorders: implications and limitations. Journal of Neural Transmission 111: 387-411.

29. Santarelli L, Saxe M, Gross C, Surget A, Battaglia F, et al. (2003) Requirement of hippocampal neurogenesis antidepressant treatments and animal models of depressive like behaviour. Behavioural Pharmacology 301: 805-809.

30. Wiltrout C, Lang B, Yan Y, Dempsey RJ, Vemuganti R (2007) Repairing brain after stroke: a review on post-ischemic neurogenesis. Neurochem Int 50: 1028-1041.
31. Taupin P (2006) Adult neurogenesis and neuroplasticity. Restor Neurol Neurosci 24: 9-15.

32. Lim CM, Kim WW, Park JY, Kim C, Yoon SH, et al. (2009) Fluoxetine affords robust neuroprotection in the post-ischemic brain via its antiinflammatory effect. Journal of Neuroscience Research 87: 1037-1045.

33. Shin TK, Kang MS, Lee HY, Seo MS, Kim SG, et al. (2009) Fluoxetine and sertraline attenuate post-ischemic brain injury in mice. Korean Journal of Physiology and Pharmacology 13: 257-263.

34. Pälvimäki EP, Laakso A, Kuoppamäki M, Syvälahti E, Hietala J (1994) Up-regulation of beta 1-adrenergic receptors in rat brain after chronic citalopram and fluoxetine treatments. Psychopharmacology (Berl) 115: 543-546.

35. Schambra H, Im B, O'Dell MW (2015) Should This Patient With Ischemic Stroke Receive Fluoxetine? PM R 7: 1294-1299. 